\title{
The effect of error propagation in the 3D reconstruction of coronary segments using CTCA images on crucial hemodynamic parameters
}

\author{
Panagiotis K. Siogkas, Antonis I. Sakellarios, Savvas K. Kyriakidis, Constantinos D. \\ Anagnostopoulos, Gualtiero Pelosi, Silvia Rocchiccioli, Lampros K. Michalis and Dimitrios I. Fotiadis
}

\begin{abstract}
The development of 3D reconstruction methods of the coronary vasculature has gained substantial ground during the past years. The accurate $3 \mathrm{D}$ reconstruction is of utmost importance because the propagation of errors caused by either equipment calibration errors, human errors or other error sources can seriously affect the computation of critical hemodynamic parameters such as Endothelial Shear Stress, intracoronary pressures etc. In this work, we present a study on how the 3D reconstruction error can affect the subsequent blood flow simulations in 3D coronary arterial models. Eight arterial segments were reconstructed, creating the control models and were then modified in order to create an underestimated and an overestimated model of the same segment using a 5\% error. Cross-sectional ESS values, as well as, smartFFR values were calculated to examine the effect of the reconstruction error. As it was expected, the underestimated models presented with higher ESS values and lower smartFFR values, whereas the overestimated models presented with lower ESS values and higher smartFFR values, respectively.
\end{abstract}

\section{INTRODUCTION}

Due to the continuously increasing rate of mortality caused by cardiovascular diseases, the interest in the development of invasive or non-invasive techniques for the hemodynamic assessment of coronary stenoses has gained substantial ground during the past two decades. Coronary imaging modalities such as Invasive Coronary Angiography (ICA), IntraVascular UltraSound (IVUS), Computed Tomography Coronary Angiography (CTCA) are used in everyday clinical practice and the choice of the exam of preference relies mainly on other clinical findings or symptoms. However, during the past years, non-invasive imaging modalities have started to surpass other widely used invasive modalities due to their non-invasive nature. In particular, CTCA is now one of the most commonly used cardiac imaging modalities since it provides a visual perspective of the whole coronary vasculature and many CTCA-derived 3D reconstruction methods have already been reported in the literature $[1,2]$.

Every imaging modality however, suffers from several flaws due to either equipment imperfections, errors due to human interaction or even poor calibration of the imaging

* This work is part-funded by the European Commission. SMARTool simulation modelling in coronary artery disease: a tool for clinical decision support. GA 689068. This article reflects only the author's view. The Commission is not responsible for any use that may be made of the information it contains.

D.I. Fotiadis is with the Unit of Medical Technology and Intelligent Information Systems, Dept. of Materials Science and Engineering, University of Ioannina, GR 45110.

C.D. Anagnostopoulos is with the Center for Experimental Surgery, Clinical and Translational Research, Biomedical Research Foundation, Academy of Athens, Greece. equipment. The errors that are generated from these factors are then propagated to the reconstruction algorithms that in turn, generate 3D models of the artery of interest with a certain error included. This issue is a non-trivial matter in cardiovascular modelling because the initial error that derives from the imaging equipment is propagated in all the remaining modelling steps and could alter the final decision regarding the treatment process [3].

The gold standard in the functional assessment of coronary stenoses is the Fractional Flow Reserve (FFR), which is measured in patients undergoing ICA. Briefly, FFR is the ratio of the pressure after the stenosis of interest divided by the mean aortic pressure. FFR is measured under the pharmacological induction of hyperemia using a common vasodilator (adenosine). A dedicated pressure wire is used to measure the pressures of interest. The FFR cut-off value is 0.80 , under which, a percutaneous coronary intervention is required.

The ongoing advances in Computational Fluid Dynamics (CFD) and the application of CFD on 3D models of the coronary vasculature have allowed for the accurate and reliable computation of very important hemodynamic parameters such as Endothelial Shear Stress (ESS) and intracoronary pressures. Several studies are reported in the current literature, that focus on the development and progression of atherosclerosis or the development of invasive or non-invasive methods for the functional assessment of coronary stenoses [4-12].

In this work, we present a methodology to study the error propagation deriving from the $3 \mathrm{D}$ reconstruction of coronary arterial segments from CTCA images and how this error affects the produced results from blood flow simulations that are applied on the generated 3D models. Briefly, the correct $3 \mathrm{D}$ models are either inflated or deflated by a proportion that is defined by the maximum reconstruction error, indicating an overestimation or an underestimation of the reconstructed arterial lumen. ESS values and smartFFR values are calculated and compared in order to study the effect of the reconstruction

L.K. Michalis is with the Michaelideion Cardiac Center, Dept. of Cardiology in Medical School, University of Ioannina, GR 45110 Ioannina, Greece.

G. Pelosi and S. Rocchiccioli are with the Institute of Clinical Physiology, CNR, Pisa, Italy.

P.K Siogkas, A.I. Sakellarios, S.K. Kyriakidis and D.I. Fotiadis, are with the Dept. of Biomedical Research, FORTH-IMBB, GR 45110 Ioannina, Greece (corresponding author phone: +302651007837 ; fax: +302651008889; e-mail: fotiadis@cc.uoi.gr). 
error on the crucial hemodynamic factors that are used to assess the hemodynamic status of the artery of interest.

\section{MATERIALS AND METHODS}

\section{A. Dataset}

A group of eight patients that underwent CTCA analysis (64-slice General Electric Medical Systems Discovery PET/CT 690® scanner) was included in the current study.

\section{B. 3D Reconstruction}

The $3 \mathrm{D}$ reconstruction of the eight arterial segments was performed using our in-house developed 3D reconstruction algorithm [13]. Briefly, our algorithm is based on the following approach:

a. the CCTA images are pre-processed using the Frangi Vesselness filter.

b. The image distortion caused by intense calcifications (i.e. blooming effect), is removed.

c. The 3D centerline of the vessels is extracted by implementing a minimum cost path approach.

d. Using a function of Hounsfield Units (HU) values and the distance from the centerline, an estimation of the weight function for lumen, outer wall and calcified plaque is made.

e. A final refinement of active contour models for lumen and outer wall segmentation is implemented.

f. A level set method is applied regarding the atheromatic plaque segmentation, taking into account calcified objects of significant size.

g. Finally, the 3D surfaces for the lumen, outer wall and calcified plaques are created. For the purposes of the current study, only the arterial lumen was extracted and used.

\section{Error generation}

In order to create the $3 \mathrm{D}$ models that include the reconstruction error, we manually edited each of the eight correctly reconstructed arterial models and we created two separate 3D models which included either an overestimation of the arterial lumen (i.e. $5 \%$ diameter increase over the average diameter of the initial model) or an underestimation of the arterial lumen (i.e. 5\% deflation), thus simulating the possible maximum reconstruction error of $5 \%$ (Fig. 1).

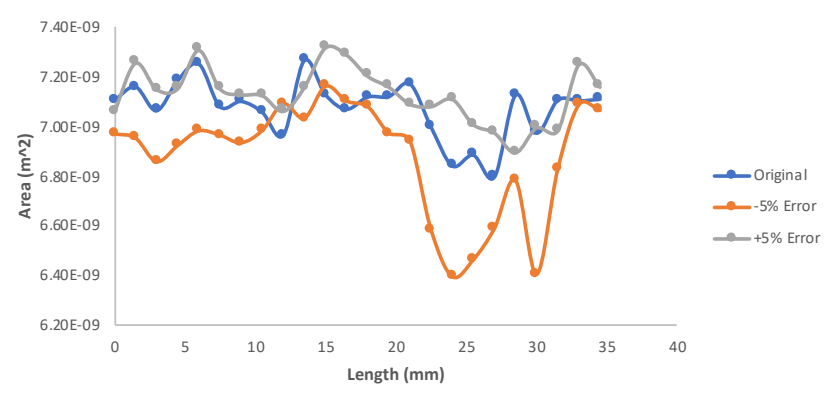

Fig. 1: Cross-sectional area of an indicative case for all 3 models.

\section{Blood Flow Simulations}

In order to examine the effect of the error in the reconstructed vessels, our scope was to calculate the average ESS in cross-sections that had a distance of $1.5 \mathrm{~mm}$ between them for each case (i.e. three different models), using the finite element method (FEM). Moreover, we calculated the smartFFR index for all cases to study how the error affected the hemodynamic significance of the stenoses that were present.

\section{a. Rigid Wall Assumption}

In order to model blood flow in our simulations, we used the Navier-Stokes and the continuity equations:

$$
\begin{aligned}
& \rho \frac{\partial \mathbf{v}}{\partial t}+\rho(\mathbf{v} \bullet \nabla) \mathbf{v}-\nabla \bullet \boldsymbol{\tau}=0, \\
& \nabla \bullet(\rho \mathbf{v})=0
\end{aligned}
$$

where $\mathbf{v}$ is the blood velocity vector and $\boldsymbol{\tau}$ is the stress tensor, which is defined as:

$$
\tau=-p \delta_{i j}+2 \mu \varepsilon_{i j}
$$

where $\delta_{i j}$ is the Kronecker delta, $\mu$ is the blood dynamic viscosity, $p$ is the blood pressure and $\varepsilon_{i j}$ is the strain tensor calculated as:

$\varepsilon_{i j}=\frac{1}{2}\left(\nabla \mathbf{v}+\nabla \mathbf{v}^{T}\right)$,

Blood was treated as a Newtonian fluid, with density 1050 $\mathrm{kg} / \mathrm{m}^{3}$ and dynamic viscosity $0.0035 \mathrm{~Pa} \cdot \mathrm{s}$. The generated flow was considered laminar and incompressible. The Reynolds number ranged from 245-1267.

\section{b. Boundary Conditions}

To calculate the smartFFR index, one transient blood flow simulation was carried out for all the examined cases. Regarding the inlet, an average pressure of $100 \mathrm{mmHg}$ was used in all simulations (i.e. mean human aortic pressure). At the outlet, a transient flow simulation is carried out, consisting of 5 timesteps with timestep duration of $0.25 \mathrm{sec}$. In each timestep, a volumetric flow rate of $0,1,2,3$ and $4 \mathrm{ml} / \mathrm{s}$ is applied as outlet boundary conditions, respectively. Regarding the arterial wall, no-slip and no-penetration boundary conditions are used. In order to build the patient-specific $\mathrm{P}_{d} / \mathrm{P}_{\mathrm{a}}$ curve, we calculated the $\mathrm{P}_{\mathrm{d}} / \mathrm{P}_{\mathrm{a}}$ value for every timestep of the simulation and the final values are then plotted and fitted by a smoothing spline consisting of 100 points. The final curve is then used to calculate the area under the curve which is then normalized by dividing by the AUC of the same artery if no pressure drop was observed (i.e. healthy segment). The calculated ratio represents the smartFFR value. Regarding the ESS values, we used the results that were produced for a flow of $1 \mathrm{ml} / \mathrm{s}$, which corresponds to the average blood flow during rest.

\section{c. Mesh}

All 3D models were discretized into tetrahedral elements using identical meshing parameters, with an element face size ranging between 0.12 and $0.14 \mathrm{~mm}$ resulting to a total mesh size of around one million elements. The element size was determined after a mesh sensitivity analysis. We used identical meshing parameters in order to eliminate any deviations in the final results due to mesh differences. The limit of iterations for the convergence of the analysis was 50 and the convergence criterion was $10^{-4}$. 


\section{RESULTS}

In order to determine the extent of the effect of the error propagation from the $3 \mathrm{D}$ reconstruction process to the final simulation results, we calculated the mean ESS in crosssections that had a distance $1.5 \mathrm{~mm}$ for each 3D model. In each of the eight cases, the cross-sections were created using the centerline that was extracted from the initial 3D model. The centerline remained intact since the length of each case remained the same for both error-including 3D models, thus meaning that the same locations were examined for every triplet of models. Regression plots were created to compare the overestimated models to the correct ones, as well as the underestimated models to the correct ones, respectively. The Bland-Altman method of analysis was also used to examine the agreement between the correctly reconstructed models and the error-including ones.

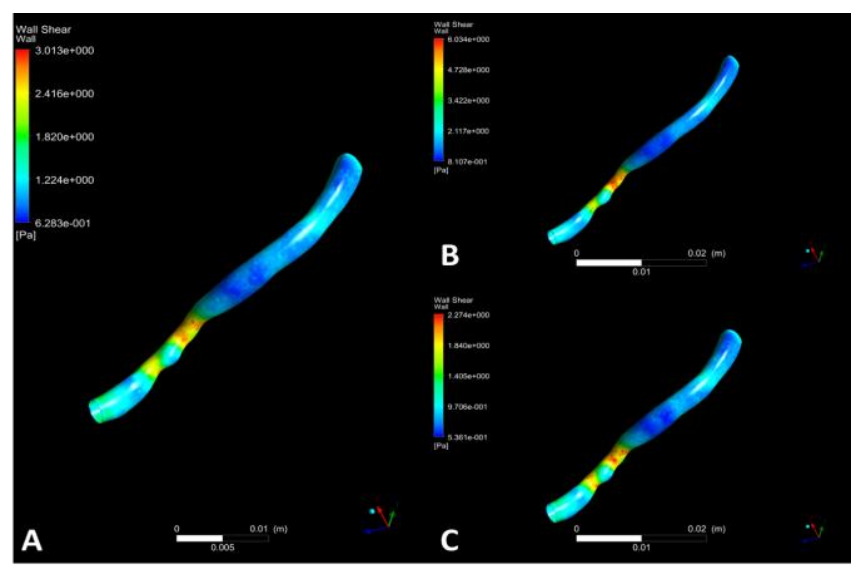

Fig. 2: A) ESS distribution for the original 3D model, B) ESS distribution for the underestimated lumen model and C) ESS distribution for the overestimated lumen model.

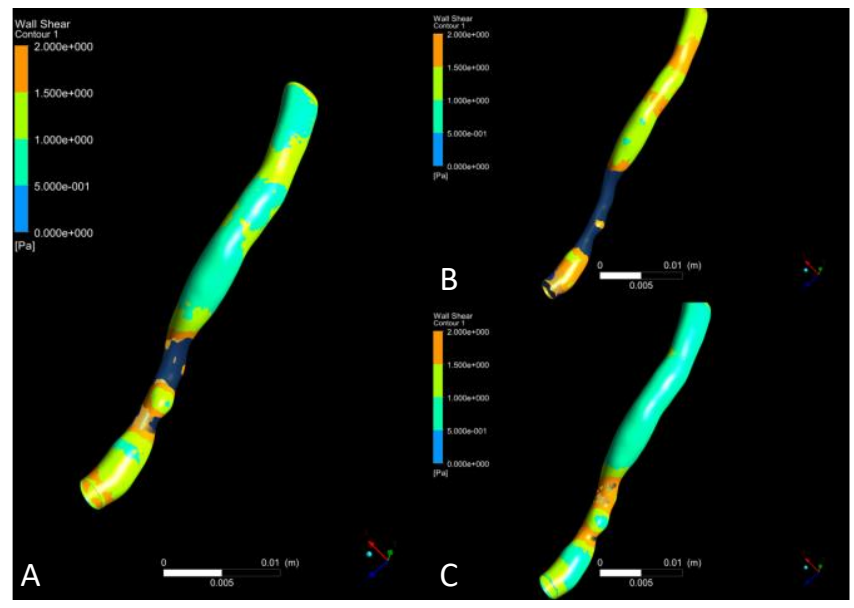

Fig. 3: Areas of low ESS for: A) original 3D reconstructed model, B) underestimated lumen model, and C) overestimated lumen model.

Strong correlation is observed between the correct 3D models and the underestimated models in the ESS calculations $(\mathrm{R}=0.99, \mathrm{p}<0.0001)$ (Fig. 4), whereas a moderate correlation is found between the original $3 \mathrm{D}$ models against the overestimated ones $(\mathrm{R}=0.83$, $\mathrm{p}<0.0001)$ (Fig. 6). Good agreement is found for the underestimated models with a mean difference of $2.56 \mathrm{~Pa}$ compared to the original ones, as depicted by the Bland-Altman method of analysis (Fig. 5).
Regarding the overestimated models, good agreement was also found with a mean difference $1.24 \mathrm{~Pa}$ compared to the original 3D models (Fig. 7).

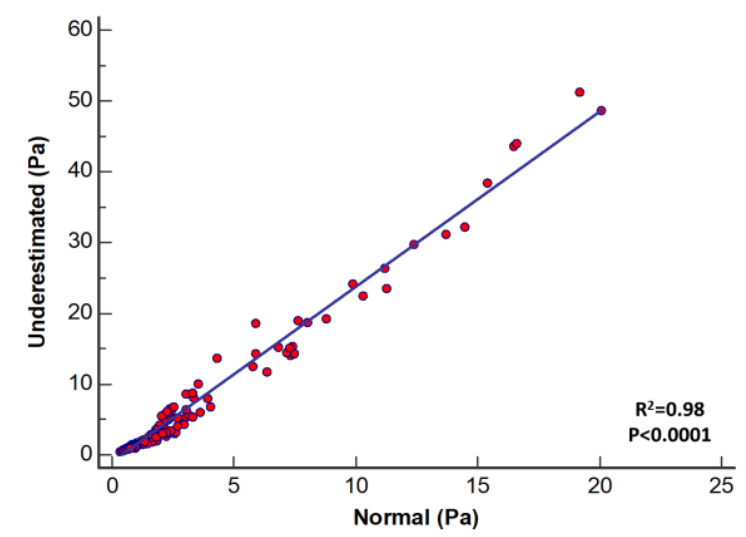

Fig. 4: Regression plot comparing the cross-sectional ESS between the original and the underestimated models.

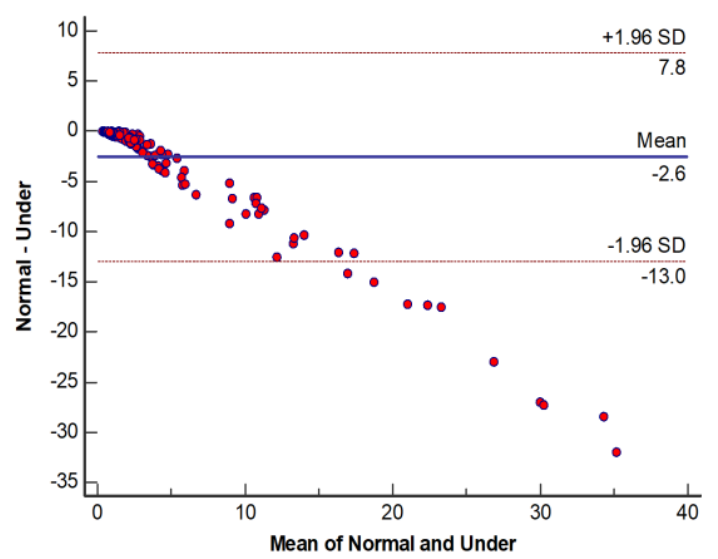

Fig. 5: Bland-Altman plot comparing the cross-sectional ESS between the original and the underestimated models.

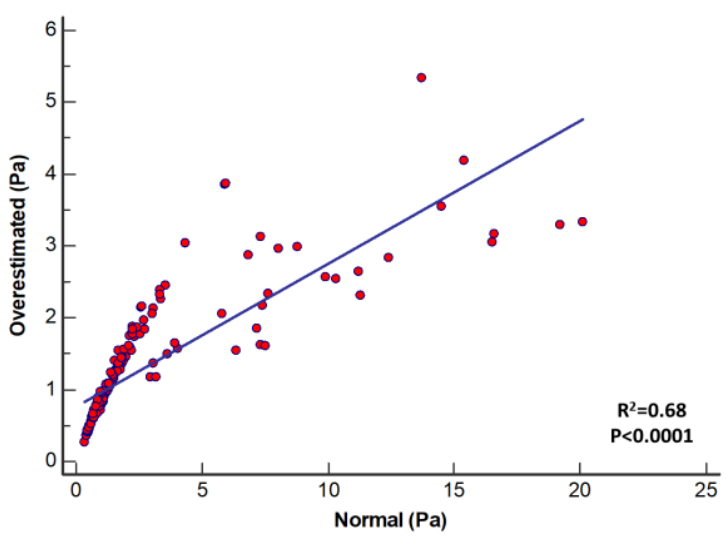

Fig. 6: Regression plot comparing the cross-sectional ESS between the original and the overestimated models.

Regarding the smartFFR values, when dealing with unobstructed arterial segments, as it was clearly expected, the underestimated lumen models presented with lower smartFFR values, whereas the overestimated lumen models presented with higher values (Table I). 


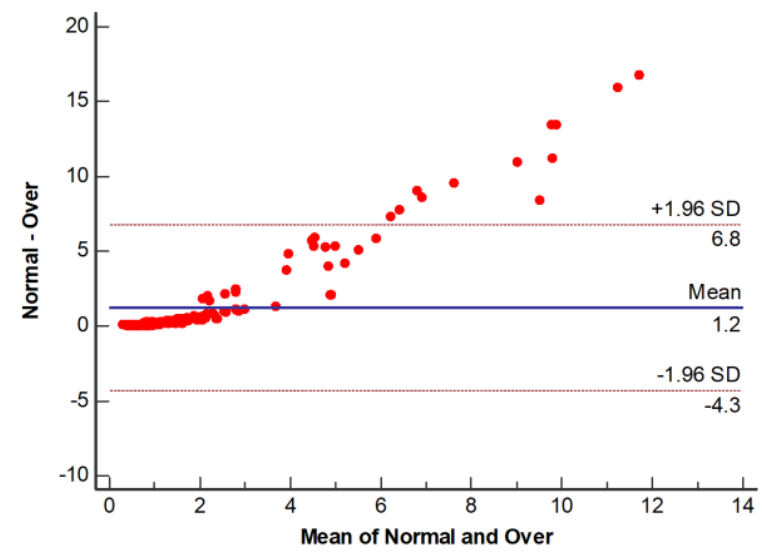

Fig. 7: Bland-Altman plot comparing the cross-sectional ESS between the original and the overestimated models.

TABLE I. CALCULATED SMARTFFR FOR THE INITIAL 3D MODELS VERSUS THE OVERESTIMATED AND UNDERESTIMATED LUMEN MODELS

\begin{tabular}{cccc}
\hline Case \# & Original & $\begin{array}{c}\mathbf{- 5 \%} \\
\text { Error }\end{array}$ & $\mathbf{+ 5 \%}$ Error \\
\hline $\mathbf{1}$ & 0.96 & 0.93 & 0.98 \\
$\mathbf{2}$ & 0.96 & 0.94 & 0.98 \\
$\mathbf{3}$ & 0.71 & 0.67 & 0.76 \\
$\mathbf{4}$ & 0.98 & 0.96 & 0.99 \\
$\mathbf{5}$ & 0.99 & 0.98 & 0.99 \\
$\mathbf{6}$ & 0.93 & 0.85 & 0.96 \\
$\mathbf{7}$ & 0.95 & 0.9 & 0.97 \\
$\mathbf{8}$ & 0.91 & 0.87 & 0.94 \\
\hline
\end{tabular}

\section{DISCUSSION}

In this work we presented an error propagation study and its effect on the calculation of critical hemodynamic parameters such as ESS and intracoronary pressures. Eight arterial segments were initially reconstructed in 3D using CTCA images and for each case we created two additional models, one depicting an overestimation of the diameter of the arterial lumen and one depicting an underestimation, both by $5 \%$. The calculation of the ESS values revealed similar trends for both cases, with the underestimated models presenting with a high correlation coefficient value and the overestimated models presenting with a more modest result. We must here state that, the areas of low ESS values (0-2 Pa) cover a larger portion of the vessel in the overestimated model and a significantly smaller portion in the underestimated lumen model, compared to the original one (Fig. 2, Fig. 3). We can also observe that smartFFR is also affected by the reconstruction error, without however being affected to such an extent that would produce a false classification of the examined case (i.e. healthy or ischemic). In particular, the only pathological case of our dataset was correctly classified as such by both the over- and the underestimated model. The healthy segments were correctly assessed as healthy by both the over- and the underestimated models. The limitation of our study was the modest sample size, which however, allowed for the extraction of useful conclusions emphasizing the importance of an accurate 3D reconstruction of coronary arteries.

\section{CONCLUSIONS}

The error that can be generated and propagated throughout the entire 3D reconstruction process workflow regarding CTCA-derived 3D models, is a non-trivial matter which can significantly alter the calculated blood flow simulation results and subsequently mislead the clinician for the final decisionmaking process.

\section{REFERENCES}

J. Menke, C. Unterberg-Buchwald, W. Staab, J. M. Sohns, A. Seif Amir Hosseini, and A. Schwarz, "Head-to-head comparison of prospectively triggered vs retrospectively gated coronary computed tomography angiography: Meta-analysis of diagnostic accuracy, image quality, and radiation dose," Am Heart J, vol. 165, no. 2, pp. 154-63 e3, Feb 2013.

[2] M. M. Vorre and J. Abdulla, "Diagnostic accuracy and radiation dose of CT coronary angiography in atrial fibrillation: systematic review and meta-analysis," Radiology, vol. 267, no. 2, pp. 37686, May 2013.

[3] L. S. Athanasiou et al., "Error propagation in the characterization of atheromatic plaque types based on imaging," Comput Methods Programs Biomed, vol. 121, no. 3, pp. 161-74, Oct 2015.

[4] M. Renker et al., "Comparison of diagnostic value of a novel noninvasive coronary computed tomography angiography method versus standard coronary angiography for assessing fractional flow reserve," Am J Cardiol, vol. 114, no. 9, pp. 1303-8, Nov 1 2014.

[5] M. Kruk et al., "Workstation-Based Calculation of CTA-Based FFR for Intermediate Stenosis," JACC Cardiovasc Imaging, vol. 9, no. 6, pp. 690-9, Jun 2016.

[6] B. L. Norgaard et al., "Diagnostic performance of noninvasive fractional flow reserve derived from coronary computed tomography angiography in suspected coronary artery disease: the NXT trial (Analysis of Coronary Blood Flow Using CT Angiography: Next Steps)," J Am Coll Cardiol, vol. 63, no. 12, pp. 1145-1155, Apr 12014.

[7] B. S. Ko et al., "Diagnostic Performance of Transluminal Attenuation Gradient and Noninvasive Fractional Flow Reserve Derived from 320-Detector Row CT Angiography to Diagnose Hemodynamically Significant Coronary Stenosis: An NXT Substudy," Radiology, vol. 279, no. 1, pp. 75-83, Apr 2016.

[8] P. S. Douglas et al., "Clinical outcomes of fractional flow reserve by computed tomographic angiography-guided diagnostic strategies vs. usual care in patients with suspected coronary artery disease: the prospective longitudinal trial of FFR(CT): outcome and resource impacts study," Eur Heart J, vol. 36, no. 47, pp. 3359-67, Dec 142015.

[9] P. K. Siogkas et al., "Noninvasive CT-based hemodynamic assessment of coronary lesions derived from fast computational analysis: a comparison against fractional flow reserve," Eur Radiol, Oct 152018.

[10] C. D. Anagnostopoulos et al., "Characterization of functionally significant coronary artery disease by a coronary computed tomography angiography-based index: a comparison with positron emission tomography," Eur Heart $J$ Cardiovasc Imaging, Jan 92019.

[11] S. Gaur et al., "FFR Derived From Coronary CT Angiography in Nonculprit Lesions of Patients With Recent STEMI," JACC Cardiovasc Imaging, vol. 10, no. 4, pp. 424-433, Apr 2017.

[12] B. E. Stahli et al., "Comparison of resting distal to aortic coronary pressure with angiography-based quantitative flow ratio," Int J Cardiol, vol. 279, pp. 12-17, Mar 152019.

[13] A. I. Sakellarios et al., "SMARTool: A tool for clinical decision support for the management of patients with coronary artery disease based on modeling of atherosclerotic plaque process," Conf Proc IEEE Eng Med Biol Soc, vol. 2017, pp. 96-99, Jul 2017. 